Publications of the Astronomical Society of the Pacific, 115:807-810, 2003 July

(C) 2003. The Astronomical Society of the Pacific. All rights reserved. Printed in U.S.A.

\title{
Rotational Velocities of B, A, and Early-F Narrow-lined Stars
}

\author{
FranCIS C. FeKeL ${ }^{1}$ \\ Center of Excellence in Information Systems, Tennessee State University, 330 10th Avenue North, Nashville, TN 37203 \\ Received 2003 March 27; accepted 2003 April 7
}

\begin{abstract}
Projected rotational velocities for $58 \mathrm{~B}, \mathrm{~A}$, and early-F stars have been determined from highresolution spectroscopic observations made at Kitt Peak National Observatory with the coudé feed telescope. All the stars are slowly rotating with $v \sin i<60 \mathrm{~km} \mathrm{~s}^{-1}$. Because of their low rotational velocities, 15 of the stars have been observed as prospective, early-type, radial velocity standards.
\end{abstract}

\section{INTRODUCTION}

The projected rotational velocity of a star is a basic datum that is important in understanding the evolution of both single and binary stars. For some early-type spectral classes, rotational velocity appears to be a discriminant for stars with peculiar spectra, such as Be stars (Briot 1986) and Am and Ap stars (Abt \& Morrell 1995). Rotational velocities can also be used to determine observationally close binary synchronization and compare the results with theory (Giuricin, Mardirossian, \& Mezzetti 1984a, 1984b; Pan 1997). Projected rotational velocity surveys of A- and F-type stars include those of Danziger \& Faber (1972), Abt \& Morrell (1995), Wolff \& Simon (1997), and Royer et al. (2002a, 2002b). Abt, Levato, \& Grosso (2002) recently measured the projected rotational velocities of nearly 1100 bright, northern B-type stars.

Over the past decade spectroscopic observations of $58 \mathrm{~B}, \mathrm{~A}$, and early F-type stars have been acquired to identify early-type constant velocity stars and to use as reference stars for spectraltype determinations. As part of the analysis, projected rotational velocities have been measured for these stars.

\section{OBSERVATIONS}

High-resolution spectroscopic observations were obtained with the Kitt Peak National Observatory (KPNO) coudé feed telescope, coudé spectrograph, and a TI CCD detector. Most of the spectrograms were centered in the red at $6430 \AA$, but additional observations were made in the blue at $4500 \AA$. The red spectra have a resolution of $0.21 \AA$, while the blue spectra have a nearly identical resolution of $0.22 \AA$. The spectra cover a wavelength range of about $80 \AA$ and have typical signal-tonoise ratios of 200. Both blue and red wavelength observations

\footnotetext{
${ }^{1}$ Visiting Astronomer, Kitt Peak National Observatory, National Optical Astronomy Observatory, operated by the Association of Universities for Research in Astronomy, Inc., under cooperative agreement with the National Science Foundation.
}

were made for 20 stars, while a single observation was obtained for 13 stars.

\section{3. $v \sin i$ MEASUREMENTS}

The projected rotational velocities have been determined from the spectra of two different wavelength regions, 6430 and $4500 \AA$. In the $6430 \AA$ region the measurable lines for $\mathrm{A}$ and early- $\mathrm{F}$ stars are primarily modest-strength $\mathrm{Fe}$ I features but also include $\mathrm{Fe}$ II and $\mathrm{Ca}$ I lines (Fig. 1). However, for B and some early-A type stars, those features are too weak for accurate determination of $v \sin i$. In the $4500 \AA$ spectra the measured lines depend on the spectral class of the star. For early$\mathrm{B}$ stars, $\mathrm{Al}$ III and $\mathrm{N}$ II lines were used, while for later spectral classes from mid-B to early-F, various $\mathrm{Fe}$ II and $\mathrm{Ti}$ II features can be measured (Fig. 2). Because they have large equivalent widths, the He I line at $4471 \AA$ and the Mg II line at $4481 \AA$ are useful lines to measure in the spectra of rapidly rotating B and A stars. However, the He I line has Stark-broadened wings and is blended with the forbidden [He I] component at $4470 \AA$ (Slettebak et al. 1975), and the Mg II line is a close doublet. Because all the stars in this work are rotating relatively slowly, neither line was measured.

The procedure of Fekel (1997) has been used to determine the $v \sin i$ values. For each star, the full width at half-maximum (FWHM) of several metal lines in the $6430 \AA$ region was determined from a Gaussian fit, and the results were averaged. An instrumental broadening of $0.21 \AA$ was removed from the measured broadening by taking the square root of the difference between the squares of measurements of the stellar and comparison lines, which resulted in the intrinsic broadening. The calibration polynomial of Fekel (1997), which is based on the results of Gray (e.g., Gray 1982, 1984, 1989), was used to convert this broadening in angstroms into a total line broadening in $\mathrm{km} \mathrm{s}^{-1}$.

Spectra centered at $4500 \AA$ were analyzed in a manner similar to that for the red-wavelength spectra. The FWHM of several metal lines was measured, the results averaged, and an instru- 


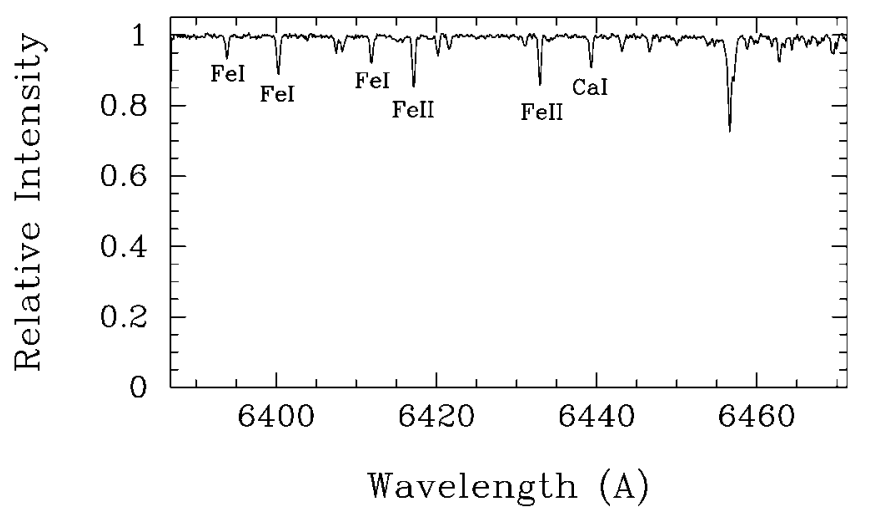

FIG. 1.-Portion of the red-wavelength spectrum of HR $1389=68$ Tauri, spectral type A2 IV-Vs. The element and ionization stage are identified for several relatively isolated lines. In this wavelength region the Fe I lines increase rapidly in strength with later spectral class.

mental broadening of $0.22 \AA$ removed. Results from the 20 stars with both blue- and red-wavelength observations were used to convert the total intrinsic stellar broadening at $4500 \AA$ to a $v \sin i$ value.

A second-order polynomial,

$$
\text { FWHM }_{4500}=0.08016+0.01284 X+0.00011 X^{2},
$$

where $X$ is the value of the total intrinsic broadening at 6430 $\AA$, was fitted to the data (Fig. 3) and used as a calibration curve. Since our blue-wavelength calibration is based on our red-wavelength results, it is also tied to the work of Gray.

Following Fekel (1997), for early-F stars, a macroturbulence of $5 \mathrm{~km} \mathrm{~s}^{-1}$ has been adopted and removed. For B and A stars, no additional broadening was removed. Uncertainties of 1 and $3 \mathrm{~km} \mathrm{~s}^{-1}$ were estimated for $v \sin i$ values near 20 and $50 \mathrm{~km}$ $\mathrm{s}^{-1}$, respectively. Table 1 lists the results for 58 stars. Spectral types are from the literature, and the early-type, radial velocity standard candidates of Fekel (1999) are identified. The $v \sin i$ determinations from the blue and red spectrograms are listed separately and also averaged if there were measurements in both wavelength regions. These values supercede the results presented by Fekel (2003), which were based in part on a preliminary blue-wavelength calibration curve.

Two early B stars, HR 153 and HR 8768, have variable line widths, which likely result from nonradial pulsations. Their $v \sin i$ values in Table 1 are followed by a " $\mathrm{v"}$ to indicate that variability.

\section{DISCUSSION}

Recently, Royer et al. (2002a, 2002b) determined $v \sin i$ values for over 750 late-B to early-F stars. Their rotational velocities were measured from the frequency of the first zero in the Fourier transforms of several line profiles. Twenty-eight of the stars in Table 1 are in common with that sample, and in

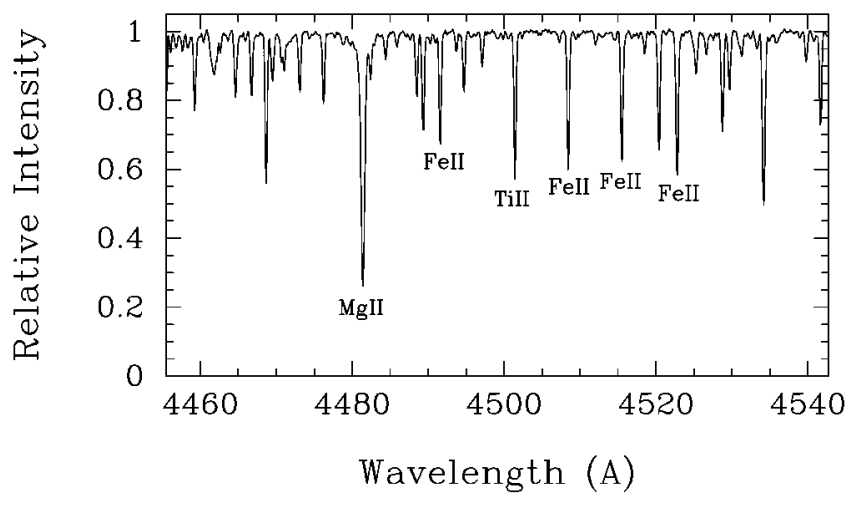

FIG. 2.-Portion of the blue-wavelength spectrum of HR $1389=68$ Tauri, spectral type A2 IV-Vs. The element and ionization stage are identified for several relatively isolated lines.

Figure 4 their individual projected rotational velocities are compared with those of this paper. Although the velocity range covered is somewhat limited, there is generally excellent agreement between our results except for the lowest and highest $v \sin i$ values, which require some explanation. The two surveys of Royer et al. (2002a, 2002b), for southern stars and northern stars, respectively, were obtained at different observatories with different spectrographs, and so the two sets of spectra have different resolutions. Royer et al. (2002b) estimated a resolution $R \approx 28,000$ at $4500 \AA$ for the southern sample and $R \approx$ 16,000 for the northern sample. The latter resolution is nearly a factor of 2 lower than that of the red-wavelength observations in this paper. Thus, at rotational velocities near $15 \mathrm{~km} \mathrm{~s}^{-1}$, the departure of the northern sample $v \sin i$ values from the oneto-one relation (Fig. 4) is caused by the limiting resolution of the spectra of Royer et al. (2002b). For the seven stars with $v \sin i \geq 35 \mathrm{~km} \mathrm{~s}^{-1}$, our velocities are systematically larger than those of Royer et al. (2002b) by an average of $1.8 \mathrm{~km} \mathrm{~s}^{-1}$. One reason for this difference may be that, as the rotational broad-

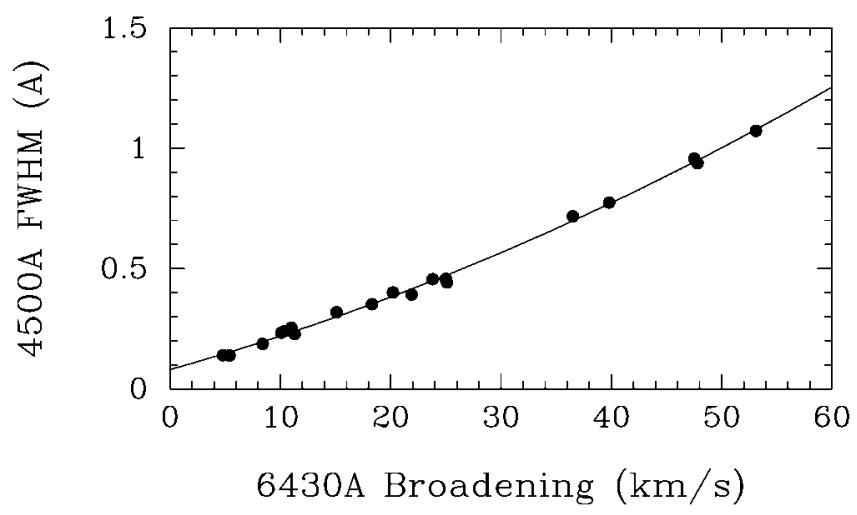

FIG. 3.-Best-fit relationship for 20 stars with both blue and red spectra. Plotted on the abscissa is the total stellar line broadening measured at 6430 $\AA$. The ordinate is the stellar FWHM measurement at $4500 \AA$. 
TABLE 1

$v \sin i$ VAlues of B, A, ANd F Stars

\begin{tabular}{|c|c|c|c|c|c|}
\hline \multirow[b]{2}{*}{ HR } & \multirow[b]{2}{*}{ HD } & \multirow{2}{*}{$\begin{array}{c}\text { SPECTRAL } \\
\text { TyPE }\end{array}$} & \multicolumn{3}{|c|}{$v \sin i^{\mathrm{a}}$} \\
\hline & & & $6430 \AA$ & $4500 \AA$ & Final \\
\hline $153^{\mathrm{b}}$ & 3360 & B2 IV & & $20.6 \mathrm{v}$ & $21 v$ \\
\hline $675^{\mathrm{b}}$ & 14252 & A2 IVs & 25.0 & 24.3 & 25 \\
\hline $811 \ldots \ldots \ldots$ & 17081 & B7 V & $\ldots$ & 21.2 & 21 \\
\hline $895 \ldots \ldots \ldots$ & 18557 & $\mathrm{~A} 2 / \mathrm{A} 6: / \mathrm{F} 0$ & 18.7 & & 19 \\
\hline $1174 \ldots \ldots \ldots$ & 23793 & B3 V & $\ldots$ & 48.4 & 48 \\
\hline $1296 \ldots \ldots \ldots$ & 26553 & $\mathrm{~A} 3 / \mathrm{A} 7 / \mathrm{A} 5$ & 6.1 & $\ldots$ & 6 \\
\hline $1389^{\mathrm{b}} \ldots \ldots \ldots$ & 27962 & A2 IV-Vs & 10.1 & 11.0 & 11 \\
\hline $1397 \ldots \ldots \ldots$ & 28114 & B6 IV & $\ldots$ & 20.4 & 20 \\
\hline $1458 \mathrm{Aa}^{\mathrm{c}} \ldots \ldots$ & 29140 & A4/A6/A7 & $\ldots$ & 39.7 & 40 \\
\hline $1613 \ldots \ldots \ldots$ & 32115 & A9 V & 11.0 & 12.2 & 12 \\
\hline $1637 \ldots \ldots \ldots$ & 32537 & $\mathrm{~F} 1 \mathrm{Vp}$ & 17.0 & $\ldots$ & 17 \\
\hline $1664 \ldots \ldots \ldots$ & 33054 & $\mathrm{~A} 2 / \mathrm{F} 2 / \mathrm{F} 3$ & 39.5 & 39.9 & 40 \\
\hline $1810^{\mathrm{b}} \ldots \ldots \ldots$ & 35708 & B2.5 IV & $\ldots$ & 29.4 & 29 \\
\hline $2010^{\mathrm{b}}$ & 38899 & B9 IV & $\ldots$ & 29.9 & 30 \\
\hline $2085 \ldots \ldots \ldots$ & 40136 & F2 IV & 16.6 & $\ldots$ & 17 \\
\hline $2124 \mathrm{Aa} \ldots \ldots$. & 40932 & $\mathrm{~A} 4 / \mathrm{A} 5 / \mathrm{A} 7$ & 9.7 & $\ldots$ & 10 \\
\hline $2154^{\mathrm{b}} \ldots \ldots \ldots$ & 41692 & B5 IV & $\ldots$ & 35.2 & 35 \\
\hline $2238 \ldots \ldots \ldots$ & 43378 & A1 Va & 47.5 & 48.3 & 48 \\
\hline $2421 \ldots \ldots \ldots$ & 47105 & A2 IV & 11.3 & 10.6 & 11 \\
\hline $2489^{\mathrm{b}} \ldots \ldots \ldots$ & 48843 & A8 II & 10.4 & 11.4 & 11 \\
\hline $2818^{\mathrm{b}}$ & 58142 & A0mA1 IV & 18.3 & 18.3 & 18 \\
\hline $3136^{\mathrm{b}}$. & 65900 & A1 IV & 36.5 & 37.5 & 37 \\
\hline $3354 \ldots \ldots \ldots$ & 72037 & $\mathrm{~A} 3 / \mathrm{A} 5 / \mathrm{A} 7$ & 11.1 & 11.2 & 11 \\
\hline $3383^{b} \ldots \ldots \ldots$ & 72660 & A1 II & 4.8 & 4.5 & 5 \\
\hline $3526 \ldots \ldots \ldots$ & 75811 & A4/A6/A7 & 11.1 & $\ldots$ & 11 \\
\hline $4033 \ldots \ldots \ldots$ & 89021 & A1 IV & $\ldots$ & 53.4 & 53 \\
\hline $4187 \ldots \ldots \ldots$ & 92728 & $\mathrm{~A} 0 \mathrm{Vs}$ & 21.9 & 20.6 & 21 \\
\hline $4237 \mathrm{~A}^{\mathrm{c}} \ldots \ldots$ & 93903 & A3/A7 V/A9 & 20.2 & 21.1 & 21 \\
\hline $4295^{\mathrm{b}} \ldots$ & 95418 & A1 IV & 47.8 & 47.5 & 48 \\
\hline $4359^{\mathrm{b}}$. & 97633 & A2 IV & 25.1 & 23.5 & 24 \\
\hline $4378 \ldots \ldots \ldots$ & 98280 & $\mathrm{~A} 2 \mathrm{Vs}$ & $\ldots$ & 10.7 & 11 \\
\hline $4454 \ldots \ldots \ldots$ & 100518 & A2/A5 III/A6 & 6.6 & $\ldots$ & 7 \\
\hline $4689 \mathrm{Aa}^{\mathrm{c}} \ldots \ldots$ & 107259 & A2 IV & 5.8 & $\ldots$ & 6 \\
\hline $4717 \ldots \ldots \ldots$ & 107966 & A3 V & 55.3 & 53.1 & 54 \\
\hline $4750 A^{c} \ldots \ldots$ & 108642 & $\mathrm{~A} 2 / \mathrm{A} 7 / \mathrm{A} 7$ & 6.9 & $\ldots$ & 7 \\
\hline $4780 \ldots \ldots \ldots$ & 109367 & A5/A7/A7 & 15.1 & 16.3 & 16 \\
\hline $5017 \ldots \ldots \ldots$ & 115604 & F3 IV & 4.1 & $\ldots$ & 4 \\
\hline $5075 \ldots \ldots \ldots$ & 117201 & $\mathrm{~F} 2 \mathrm{~V}$ & 10.1 & $\ldots$ & 10 \\
\hline $5445 \ldots \ldots \ldots$ & 128093 & F5 V & $\ldots$ & 8.1 & 8 \\
\hline $5447^{\mathrm{b}} \ldots \ldots \ldots$ & 128167 & $\mathrm{~F} 2 \mathrm{~V}$ & 7.7 & $\ldots$ & 8 \\
\hline $6035 \ldots \ldots \ldots$ & 145647 & A1 III & $\ldots$ & 46.9 & 47 \\
\hline $6041 \ldots \ldots \ldots$ & 145788 & A1 IIIs & 8.4 & 7.8 & 8 \\
\hline $6455 \ldots \ldots \ldots$ & 157087 & A3 IVs & 11.3 & $\ldots$ & 11 \\
\hline $6787 \ldots \ldots \ldots$ & 166182 & B2 IV & $\ldots$ & 44.1 & 44 \\
\hline $6844 \ldots \ldots \ldots$ & 167858 & F1 V & 7.9 & $\ldots$ & 8 \\
\hline $7287 \ldots \ldots \ldots$ & 179761 & B8 II-III & $\ldots$ & 16.6 & 17 \\
\hline $7431 \ldots \ldots \ldots$ & 184552 & A2/A7 V/F0 & 11.0 & $\ldots$ & 11 \\
\hline $7502 \ldots \ldots \ldots$ & 186377 & A6 III & 11.6 & $\ldots$ & 12 \\
\hline $7512^{\mathrm{b}}$ & 186568 & B8 III & $\ldots$ & 18.2 & 18 \\
\hline $7773^{\mathrm{b}} \ldots \ldots \ldots$ & 193432 & B9.5 Va & 23.8 & 24.2 & 24 \\
\hline $7878 \ldots \ldots \ldots$ & 196426 & B8 IIIp & $\ldots$ & 4.0 & 4 \\
\hline $7891 \ldots \ldots \ldots$ & 196724 & A0 IV & $\ldots$ & 54.0 & 54 \\
\hline $7903 \ldots \ldots \ldots$ & 196821 & A0 IIIps & $\ldots$ & 22.9 & 23 \\
\hline $8404^{\mathrm{b}} \ldots \ldots \ldots$ & 209459 & B9.5 V & 4.0 & $\ldots$ & 4 \\
\hline $8641 \ldots \ldots \ldots$ & 214994 & A1 IV & 5.4 & 4.4 & 5 \\
\hline $8704 A^{c} \ldots \ldots$ & 216494 & B9 III & $\ldots$ & 1.5 & 2 \\
\hline $8768 \ldots \ldots \ldots$ & 217811 & B2 V & $\ldots$ & $12.0 \mathrm{v}$ & $12 \mathrm{v}$ \\
\hline $8822 \ldots \ldots \ldots$ & 218753 & $\mathrm{~A} 5 / \mathrm{A} 7 / \mathrm{F} 0$ & 4.7 & $\ldots$ & 5 \\
\hline
\end{tabular}

${ }^{\mathrm{a}}$ In units of $\mathrm{km} \mathrm{s}^{-1} \cdot \mathrm{v}=$ variable line broadening.

${ }^{\mathrm{b}}$ Early-type radial velocity standard candidate (Fekel 1999).

${ }^{\mathrm{c}}$ Primary of a double-lined spectroscopic binary.

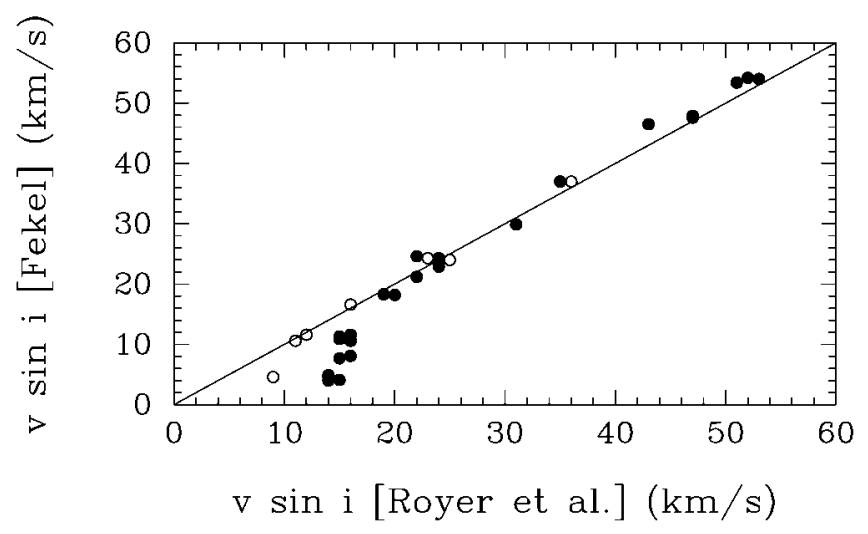

Fig. 4.-Comparison of the present data with the $v \sin i$ data of Royer et al. (2002a, 2002b). Their southern data are open circles, and their northern data are filled circles. The line showing a one-to-one correlation is plotted as a guide and is not a fit to the data.

ening of the stellar lines increases, a Gaussian curve becomes a poorer fit to the profiles. However, such a modest mean difference between our results and those of Royer et al. (2002b) is within the uncertainty estimates of our individual $v \sin i$ values.

Forty of the stars in Table 1 are in common with those measured by Abt \& Morrell (1995). Their results are based on the $v \sin i$ standard-star system of Slettebak et al. (1975), and they estimated that they could not resolve rotational velocities $\lesssim 10 \mathrm{~km} \mathrm{~s}^{-1}$. As seen in Figure 5, their $v \sin i$ values between 10 and $20 \mathrm{~km} \mathrm{~s}^{-1}$ appear to scatter substantially about our values, and some of the individual values differ from ours by $10 \mathrm{~km} \mathrm{~s}^{-1}$ or more. This suggests that the lower limit to their $v \sin i$ values is closer to 20 rather than $10 \mathrm{~km} \mathrm{~s}^{-1}$. Above the threshold value of $\sim 20 \mathrm{~km} \mathrm{~s}^{-1}$, their values are systematically too small, a result already noted by Royer et al. (2002b).

I thank F. Royer and G. Henry for commenting on an earlier

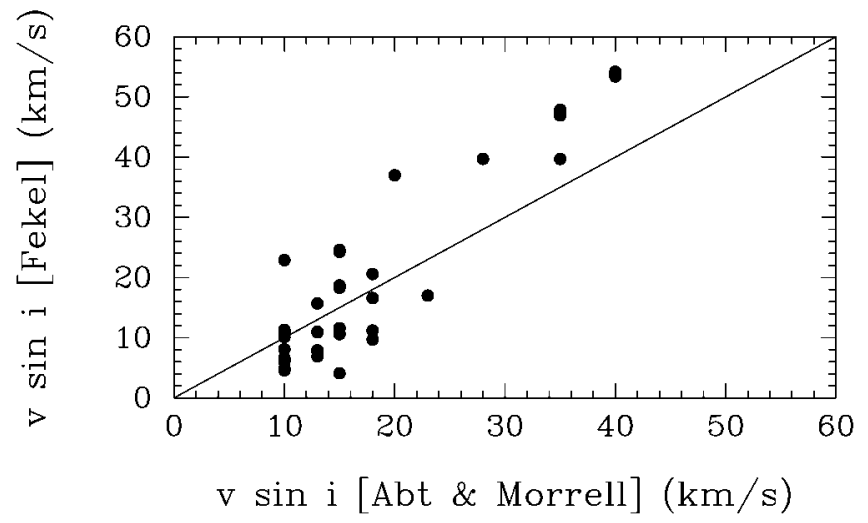

FIG. 5.-Comparison of the present data with the $v \sin i$ data of Abt \& Morrell (1995). The line showing a one-to-one correlation is plotted as a guide and is not a fit to the data. 


\section{FEKEL}

version of this work. I thank $\mathrm{H}$. Abt for his suggestions and comments. The research at Tennessee State University is sup- ported in part by NASA grant NCC5-511 and NSF grant HRD-9706268.

\section{REFERENCES}

Abt, H. A., Levato, H., \& Grosso, M. 2002, ApJ, 573, 359

Abt, H. A., \& Morrell, N. I. 1995, ApJS, 99, 135

Briot, D. 1986, A\&A, 163, 67

Danziger, I. J., \& Faber, S. M. 1972, A\&A, 18, 428

Fekel, F. C. 1997, PASP, 109, 514

1999, in ASP Conf. Ser. 185, Precise Stellar Radial Velocities, ed. J. B. Hearnshaw \& C. D. Scarfe (IAU Colloq. 170; San Francisco: ASP), 378

. 2003, in IAU Symp. 215, Stellar Rotation, ed. A. Maeder \& P. Eenens (San Francisco: ASP), in press

Giuricin, G., Mardirossian, F., \& Mezzetti, M. 1984a, A\&A, 131, 152 1984b, A\&A, 135, 393
Gray, D. F. 1982, ApJ, 258, 201

$$
\text { 1984, ApJ, 281, } 719
$$$$
\text { 1989, ApJ, 347, } 1021
$$

Pan, K. 1997, A\&A, 321, 202

Royer, F., Gerbaldi, M., Faraggiani, R. \& Gómez, A. E. 2002a, A\&A, 381,105

Royer, F., Grenier, S., Baylac, M.-O., Gómez, A. E., \& Zorec, J. 2002b, A\&A, 393, 897

Slettebak, A., Collins, G. W., II, Boyce, P. B., White, N. M., \& Parkinson, T. D. 1975, ApJS, 29, 137

Wolff, S. C., \& Simon, T. 1997, PASP, 109, 759 